\title{
Diversity of anti-haemostatic proteins in the salivary glands of Rhodnius species transmitters of Chagas disease in the greater Amazon
}

\author{
Ana C.M. Bussacos ${ }^{a, 1}$, Ernesto S. Nakayasu ${ }^{b, 1,2}$, Mariana M. Hecht ${ }^{a}$, Juliana A. Parente ${ }^{c}$, \\ Célia M.A. Soares ${ }^{c}$, Antônio R.L. Teixeira ${ }^{a, *}$, Igor C. Almeida $a^{b, * *}$ \\ ${ }^{a}$ Chagas Disease Multidisciplinary Research Laboratory, Faculty of Medicine, University of Brasília, Federal District, Brazil \\ ${ }^{\mathrm{b}}$ The Border Biomedical Research Center, Department of Biological Sciences, University of Texas at El Paso, El Paso, Texas, USA \\ ${ }^{\mathrm{c}}$ Federal University of Goiás, Goiânia, Brazil
}

\section{A R T I C L E I N F O}

Article history:

Received 9 May 2011

Accepted 21 June 2011

Available online 1 July 2011

Keywords:

Haematophagous insects

Salivary glands

Transcriptomics

Proteomics

\begin{abstract}
A B S T R A C T
The triatomines in the tribe Rhodniini are the main vectors of the Trypanosoma cruzi to humans in recent outbreaks of acute Chagas disease in the Amazon. These insects dwelling in palm trees do not colonize the human domicile. Their success to transmit the infection relies partially on the efficacy of their salivary gland apparatuses. Here we show the transcriptome of the Rhodnius brethesi and Rhodnius robustus salivary glands, comprising 56 and 122 clusters, respectively. Approximately one third of these clusters are described for the first time. The LC-MS/MS analysis identified 123 and 111 proteins in R. brethesi and $R$. robustus sialome, respectively. Noteworthy, lipocalin platelet aggregation inhibitors, inositol polyphosphate 5-phosphatases, and Kazal domain proteins, which are essential for the insect's successful acquisition of blood meals, were found in our analysis. Moreover, glutathione S transferase and antigen-5, which play roles in the insect's defense and resistance against insecticide, were also observed.
\end{abstract}

@ 2011 Elsevier B.V. All rights reserved.

\section{Introduction}

The Greater Amazon is known to hide countless dwellings of triatomines (Insecta: Hemiptera) transmitters of T. cruzi to mammals [1]. The insects preying upon over thousand mammalian species generate a huge enzooty [2] and epidemic human Chagas disease. In the past five decades increasing deforestation, new population settlements, and demographic growth in the Amazon region have concurred with autochtonous acute cases of human T. cruzi infections [3,4]. In the past years there were several outbreaks of human Chagas disease in the Brazilian Amazon Basin, where T. cruzi infections are diagnosed by the clinical manifestations of an acute ailment, or the flagellate protozoan is recognized during the routine

Abbreviations: ACN, acetonitrile; 2-DE, two-dimensional gel electrophoresis; DTT, dithiothreitol; FA, formic acid; GST, glutathione S-transferase; LC-MS/MS, liquid chromatograpgy-tandem mass spectrometry; Rb, Rhodnius brethesi; Rr, Rhodnius robustus

* Corresponding author. Tel.: +55 613349 4987; fax: +55 6132734645

** Corresponding author. Tel.: +1 915747 6086; fax: +1 9157475808.

E-mail addresses: ateixeir@unb.br (A.R.L. Teixeira), icalmeida@utep.edu (I.C. Almeida).

${ }^{1}$ Both authors contributed equally to this study.

2 Present address: Pacific Northwest National Laboratory, Richland, WA, USA. 
microscopic exams of blood smears from patients suspected of having the malaria Plasmodium parasites [5,6].

T. cruzi infections are usually transmitted to humans through direct contamination with the metacyclic parasites eliminated in the bug's excrements [5]. Among 137 blood-sucking insects belonging to the genus Rhodnius present in the American and Asian continents, there are over 40 Rhodnius spp. that have been recognized as true family Triatominae dwelling in the Amazon biome [6]. Among the Rhodnius there are 19 species comprising two major groups distributed in separate biogeographic localizations. The archetype R. brethesi is included in the group pictipes, while $R$. robustus and R. prolixus are in the group robustus [5]. These are the main transmitters of $T$. cruzi infections in the greater Amazonian ecosystem [7].

$R$. brethesi and R. robustus have always been associated with palm trees (Leopoldinia piassaba) that serve as dwells of marsupials, rodents, bats, and primates. Currently, the closeness of human settlement to palm trees and the predation of local fauna has been associated with household's invasion by Rhodnius insects [4,7]. The strictly hematophagous male and female triatomines can attack humans under daylight to transmit T. cruzi [3]. These triatomines forming large colonies are considered well-succeeded proliferating species, because they have a sophisticated machinery capable of circumventing difficulties imposed to blood sucking by the host's haemostatic mechanisms. Several important molecules were described in the insect's saliva, such as vasodilators, anticoagulants, platelet aggregation inhibitors, and anesthetics [8].

cDNA Library and liquid chromatography-tandem mass spectrometry (LC-MS/MS) are important tools employed for improving the knowledge about pharmacologically active bioamines in the triatomines' saliva [8,9]. Transcriptome analysis revealed several important bioamines for the blood feeding that are present in the salivary glands of the R. prolixus, including redundant members of lipocalins, possibly encoded by a growing gene family [9]. In addition, salivary transcriptomes from $T$. infestans [10] and T. brasiliensis [11] showed prevalence of sequences from different lipocalin genes. The 2D-gel electrophoresis (2-DE) followed by MS analysis was also used to analyze the $T$. infestans proteome $[12,13]$, and showed that among 200 proteins, there are 34 platelet aggregation inhibitors belonging to major triabin and apyrase families. All those findings showed that the salivary glands of triatomines are replete of proteins with anticoagulant, vasodilators, platelet aggregation inhibitors, anesthetics, and modulators of the mammalian immune system [8].

This study aims at the comparative analysis of the salivary transcriptome and proteome of main Rhodnius species dwelling in the Amazon. Herein, we used cDNA and LC-MS/MS approaches to provide complementary data. Sequencing of $R$. robustus salivary glands cDNA library yielded 576 ESTs, comprising 122 clusters. The R. brethesi cDNA library yielded 427 sequences in 56 clusters. By LC-MS/MS analysis we found 125 proteins in $R$. brethesi and 111 in $R$. robustus including, respectively, 86 and 93 salivary gland proteins with recognized pharmacological functions. Moreover, in both species we identified a diversity of anti-haemostatic bioamines, which play important role in the transmission of the $T$. cruzi infections and outbreaks of acute Chagas disease in the Amazon.

\section{Materials and methods}

\subsection{Triatomines and salivary gland}

$R$. brethesi and R. robustus captured from palm trees were gifts from Dr. Aldo Valente, Evandro Chagas Institute, Belém City, Pará State, Brazil. The insects were reared in the insectarium with controlled temperature of $28^{\circ} \mathrm{C}, 70 \%$ relative humidity, and 12:12 h light/dark photoperiod. Adults were dissected 3, 5, and 7 days after a blood meal. Usually, 150 pairs of salivary glands were collected in TRIZOL (Invitrogen) for the cDNA library. For the LC-MS/MS analysis, we collected 50 pairs of salivary glands, which were punctured with a needle and centrifuged. The saliva in the supernatant was collected and lyophilized, and stored at $-80^{\circ} \mathrm{C}$ until use.

\section{2. cDNA library}

The mRNA was isolated with Micro-fast Track ${ }^{\mathrm{TM}}$ mRNA isolation (Invitrogen) and cDNA library was built with SMART cDNA library construction (Clontech) as described in $[10,14]$. The PCR assays were performed on a PTC-100 programmable thermal controller (MJ Research Inc.). The PCR condition was: $95^{\circ} \mathrm{C}$ for $1 \mathrm{~min}, 19$ cycles (R. robustus) or 22 cycles (R. brethesi) of $95^{\circ} \mathrm{C}$ for $15 \mathrm{~s}$, and $68^{\circ} \mathrm{C}$ for $6 \mathrm{~min}$. The cDNA double strand obtained was digested with proteinase $\mathrm{K}$ (Invitrogen), according to the manufacturer's instructions, and after by Sfi I restriction enzyme. The cDNA was then fractionated by size using a Chroma-spin-400 drip column (Clontech) and observed in a 1.1\% Agarose/EtBr gel. The samples were collected, ligated into a $\lambda$ TriplEx2 vector (Clontech), and packed using GigaPack Gold III (Stratagen). One microliter of $\mathrm{R}$. robustus cDNA library and $2 \mu \mathrm{l}$ of $\mathrm{R}$. brethesi cDNA library were plated on $\mathrm{LB} / \mathrm{MgSO}_{4}$ plates with $\mathrm{X}$-gal/IPTG. We obtained $90 \%$ of recombination clones in both libraries. The white colonies were randomly picked and transferred in $50 \mu \mathrm{l}$ of deionized water. To amplify the cDNA sample we used $5 \mu \mathrm{l}$ of the phages as template and the primers PT2F1 and PT2R1 based on the sequence of the vector TriplEx2 (Clontech) [14]. The PCR products were sequenced unidirectionally with PT2F3 primer and DYEnamic ET DyeTerminator Sequencing Kit (Amersham Bioscience, Piscataway, NJ, USA) using a MegaBACE 1000 sequencer (Amersham Biosciences, Little Chalfont, UK). The sequence clusters were subjected to analyses using BLAST [15], ClustalW [16], CDD [17], and TREEview [18]. Phylogenetics analyses and statistical neighbor-joining bootstrap tests of the phylogenies were done with de MEGA4 package [19]. The transcripts were translated using the BioEdit program and the resulting polypeptide sequences were subject to phylogenetics analysis [18].

\subsection{LC-MS/MS analysis}

The lyophilized saliva samples of $R$. brethesi and R. robustus were diluted in $200 \mu$ l HPLC grade water (Sigma-Aldrich). Ten microliters of each sample were sequentially subjected to reduction ( $5 \mathrm{mM}$ final concentration of dithiothreitol, DTT), alkylation of the cysteine residues $(10 \mathrm{mM}$ final concentration of iodoacetamide), and digestion with sequencing grade 
modified trypsin (Promega) in $\mathrm{NH}_{4} \mathrm{HCO}_{3}$ buffer containing urea [20]. The samples were desalted using a in-house C18-reverse phase zip-tip (POROS R2-50 resin, Applied Biosystem) built in a 200- $\mu$ l micropipette tip (Axygen), containing a fine glass-wool layer at the bottom as a frit. The microcolumn was activated with HPLC-grade methanol (Sigma-Aldrich) and equilibrated with $0.046 \%$ trifluoroacetic acid (TFA) (Sigma-Aldrich). The sample was added and the microcolumn washed two times with $0.046 \%$ TFA, and then eluted with $80 \%$ ACN/0.046\% TFA. After the desalting, the samples were submitted to a strongcation exchange (SCX) column. We used the same desalting method, except for replacing the $\mathrm{C} 18$ resin with $30 \mu \mathrm{LCX}$ resin (POROS HS50, Applied Biosystems). After equilibrating the microcolumn with $25 \%$ acetonitrile (ACN)/0.5\% formic acid (FA) $(25 \%$ ACN $/ 0.5 \%$ FA), the sample was loaded and the microcolumn washed with $100 \mu 1$ 25\% ACN/0.5\% FA. Peptides were eluted with a gradient of $\mathrm{NaCl}(0,10,20,40,60,80,100$, and 150, 200 and $500 \mathrm{mM}$ ) in 25\% ACN/0.5\% FA. The samples were dried in a vacuum centrifuge (Eppendorf), desalted, and subjected to LC-MS/MS analysis. The samples were dissolved in $10 \mu \mathrm{l} 0.05 \% \mathrm{FA}$; the peptides were separated in an 1D Plus nanoHPLC system (Eksigent), and analyzed by an LTQ XL eletrospray ionization-linear ion trap-mass spectrometer (Thermo) as described [20].

BioWorks version 3.3.1 software (Thermo) converted the raw data obtained in the LC-MS to DTA files. To analyze the MS/MS spectra we first used TurboSequest available in BioWorks package $[20,21]$ algorithm with NCBInr database (downloaded on February 14, 2007). The second analysis was made with a database containing: (i) NCBI proteins from Triatoma, Panstrongylus and Rhodnius; (ii) translated transcripts from the cDNA libraries from R. brethesi, R. robustus and from Triatoma infestans and Panstrongylus megistus (unpublished); (iii) matches from the first analysis against all NCBI; and, (iv) random proteins (100 K) to be used as false-negative. We calculated the false-positive rate which was $3.73 \%$ for $R$. brethesi and $4.2 \%$ for $R$. robustus. To obtain those results we used the following filters: distinct peptides (for exclusion of redundant hits); $\mathrm{DC} n \geq 0.1$; protein probability $\leq 1 \times 10^{-3}$; and $\mathrm{Xcorr} \geq 1.5,2.2,2.7$ and 3.0 for singly, doubly, triply and more than 3 charged peptides, respectively.

\section{Results and discussion}

\subsection{Transcriptome of salivary glands of Rhodnius spp}

We obtained 576 EST grouped in 122 clusters comprising 33 contigs (cluster with more than one sequence) and 89 singlets (only one sequence) for $R$. robustus among the salivary glands cDNA sequences. For the cDNA of R. brethesi we found 427 EST in 56 clusters (19 contigs and 37 singlets). The nucleotides sequences were analyzed with blast algorithm (Blastx and Blastn). The sequences were also translated by Bioedit program and the aminoacids were submitted to CDD analysis on the NCBI site and to ClustalW algorithm followed by Mega 4 program.

As expected, Fig. 1 shows that clusters of the housekeeping transcripts comprise $27 \%$ of the R. robustus and $12.8 \%$ of the $R$. brethesi cDNAs. Ribeiro et al. [9] observed the presence of $23.5 \%$ of housekeeping proteins in R. prolixus transcriptome, $36.4 \%$ in $\mathrm{T}$. infestans [10], and $24.4 \%$ in T. brasiliensis [11]. Here we show the $R$. robustus transcriptome contains proteins related to cellular structure (calponin, villin), DNA (helicase), energy (cytochrome c oxidase I, II and III, pyruvate dehydrogenase), and protein metabolisms (ribosomal protein, 5-aminolevulinic acid synthase, histone-lysine n-methyltransferase). Also, we show the R. brethesi transcriptome contains proteins related to
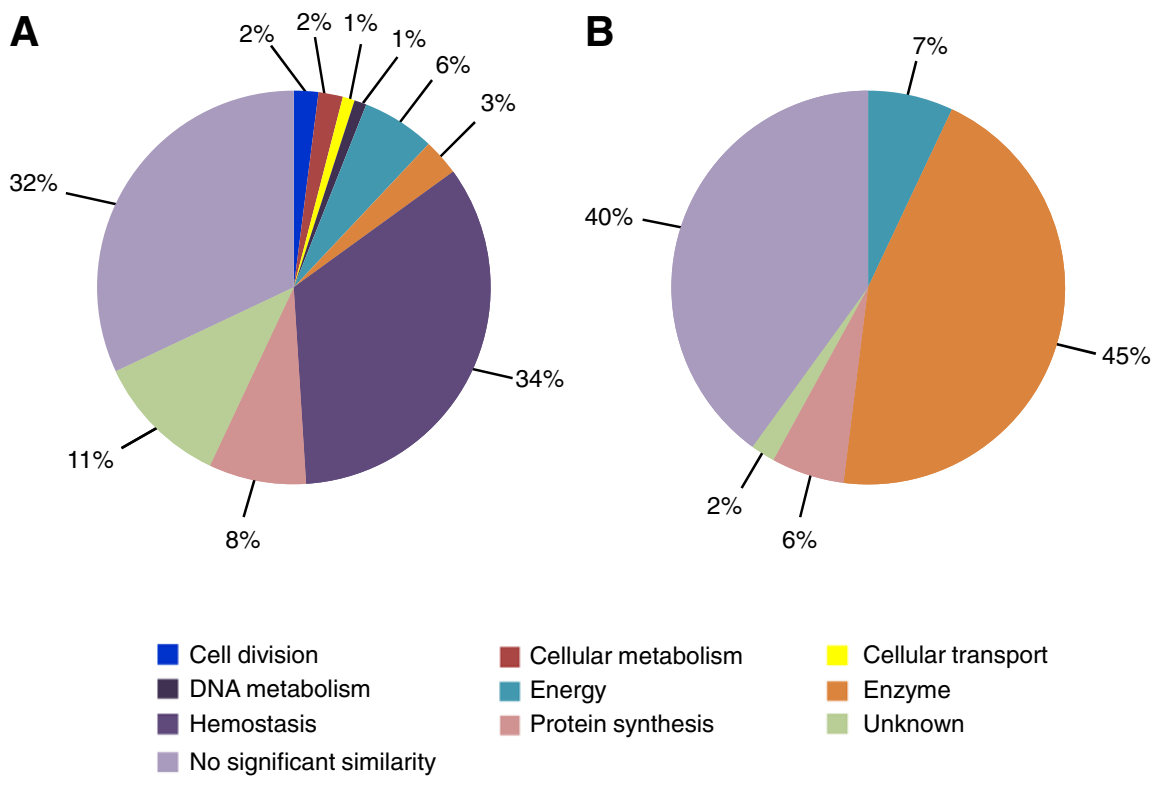

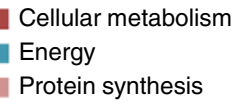

Energy

Protein synthesis
Cellular transport

Enzyme

Unknown

Fig. 1 - Potential biological function of transcripts expressed in Rhodnius robustus and Rhodnius brethesi salivary glands. A) R. robustus. B) $R$. brethesi. 
Table 1 - Rhodnius spp. proteins indispensable to the insect blood-feeding process.

\begin{tabular}{|c|c|c|c|c|}
\hline \multirow[t]{2}{*}{ Protein } & \multicolumn{2}{|c|}{ Transcriptome ${ }^{a}$} & \multicolumn{2}{|c|}{ Proteome $^{b}$} \\
\hline & R. brethesi & R. robustus & R. brethesi & R. robustus \\
\hline Antigen-5-like protein & & 1 & 4 & 2 \\
\hline Biogenic amine-binding protein & 3 & 1 & 2 & 2 \\
\hline Brasiliensin & & 1 & & \\
\hline Heme-binding protein & & & 1 & 1 \\
\hline Lipocalin & 1 & 1 & & \\
\hline Lipocalin AI-3 & 1 & 1 & 1 & 1 \\
\hline Lipocalin AI-4 & 1 & 1 & 4 & 4 \\
\hline Lipocalin AI-5 & 1 & 3 & 3 & 4 \\
\hline Lipocalin AI-6 & & 3 & 8 & 9 \\
\hline Lipocalin AI-7 & 3 & 4 & 5 & 6 \\
\hline Nitrophorin 1 & 2 & 4 & 2 & 4 \\
\hline Nitrophorin $1 \mathrm{~A}$ & 1 & 3 & 4 & 4 \\
\hline Nitrophorin 2 & 2 & 4 & 6 & 8 \\
\hline Nitrophorin 3 & 1 & 2 & 7 & 8 \\
\hline Nitrophorin $3 \mathrm{~B}$ & & 2 & 3 & 3 \\
\hline Nitrophorin 4 & & & 5 & 6 \\
\hline Nitrophorin 4A & & & 1 & 2 \\
\hline Nitrophorin 4B & 1 & 2 & 4 & 4 \\
\hline Nitrophorin 7 & & & 3 & 2 \\
\hline Pallidipin-like lipocalin 1 & & & 2 & 2 \\
\hline Pallidipin-like lipocalin 2 & 1 & 1 & & \\
\hline Polylysine protein & 1 & 1 & & \\
\hline Salivary inositol polyphosphate 5-phosphatase & & 1 & 1 & 1 \\
\hline Salivary platelet aggregation inhibitor 1 & 1 & & 1 & 1 \\
\hline Salivary platelet aggregation inhibitor 2 & & 2 & 4 & 4 \\
\hline Salivary protein MYS1 precursor & 1 & & 2 & 2 \\
\hline Triabin-like lipocalin 1 & 2 & 1 & 2 & 2 \\
\hline Triabin-like lipocalin 2 & 1 & 1 & 7 & 6 \\
\hline Triabin-like lipocalin 3 & & & 1 & 1 \\
\hline Triabin-like lipocalin 4 & & & 2 & 2 \\
\hline
\end{tabular}

energy metabolism (cytochrome c oxidase II and III, NADH dehydrogenase subunit 4, truncated ATPase subunit 6) and protein synthesis (mitochondrial ribosomal protein L30).

The most important findings in our study are proteins indispensable to the insect blood-feeding (Table 1). Biogenic amine binding proteins, lipocalins, nitrophorins, pallidipins, salivary platelet aggregation inhibitors and triabins were present in both triatomine species [23]. In our study, some transcripts that were found only in $\mathrm{R}$. robustus were brasiliensin, lipocalin AI6, nitrophorin 3B, SPAI2, and inositol polyphosphate 5-phosphatase. Differently, SPAI1 and salivary protein MYS1 precursor were found in R. brethesi only.

Several Rhodnius transcripts that matched with already sequenced proteins and were posted at NCBI database had unknown function. Moreover, these sequences appeared to be shared by Drosophila melanogaster, D. pseudoobscura, Apis mellifera, Anopheles gambiae, R. prolixus, Phlebotomus papatasi, Thermobia domestica, and Nasonia vitripennis [8,22,23]. We detected $40 \%$ of $R$. brethesi transcripts and $32 \%$ of $R$. robustus transcripts that did not match to any protein sequence in the NCBI database and, therefore, these are undisclosed proteins. Interestingly, the translated transcripts submitted to conserved domain analysis revealed that many haemostatic proteins had triabin domain (Supplementary Tables 1 and 2).

\subsection{Proteome of salivary glands of Rhodnius spp}

The proteomic analysis of Rhodnius sp salivary gland proteins convalidated several findings described in the cDNA library and depicted other not yet described. A high-throughput approach was used to generate the proteomes that were analyzed. Firstly, we matched the whole spectra of data with all NCBI databases, and selected proteins were saved for a second step where a specific database was built aiming at performing the statistical analyses. The database used retained proteins that matched translated cDNA transcripts from $R$. robustus, $R$. brethesi, P. megistus, and T. infestans proteins deposited in NCBI server, and random proteins. With this ample database we could calculate the false-positive discovery rate, as to attaining correct statistical calculations $(p<0.05)$.

A total of 111 proteins from $R$. robustus and 125 from $R$. brethesi (Supplementary Tables 3 and 4) were obtained. Among these there were $93 \mathrm{R}$. robustus proteins with pharmacological and enzymatic functions, and 86 of these proteins, which were present in the R. brethesi proteome, are shown in Table 1 . This approach secured several proteins not yet identified in cDNA libraries, such as apolipophorin, heme-binding proteins, nitrophorin 4, 4A and 7, pallidipin-like lipocalin 1, and triabin-like lipocalin 3 and 4. 


\subsubsection{Lipocalins and nitrophorins}

The lipocalins comprise a large group of small proteins (160180 residues) with different functions and tertiary structure. The recently evolved lipocalins have many substituted residues, very flexible tertiary structure, and low ligand binding and highly efficient binding rate [24]. This protein group has three possible interactions: binding to small hydrophobic proteins, binding to a receptor or forming a macromolecular complex [24]. Although there were some particular domains contrasting with lack of sequence similarity, the lipocalins formed a tertiary structure cavity to accommodate the ligand. The cavity size and aminoacids sequence are specific to each type of lipocalin and binding ligand [25]. Lipocalins can be described as Kernel proteins or outlier lipocalins [26].

Lipocalins sequences are highly diverse and their alignments are hard to achieve, and consequently a phylogenetic tree cannot be built [24-27]. Fig. 2A show some sequences from

A

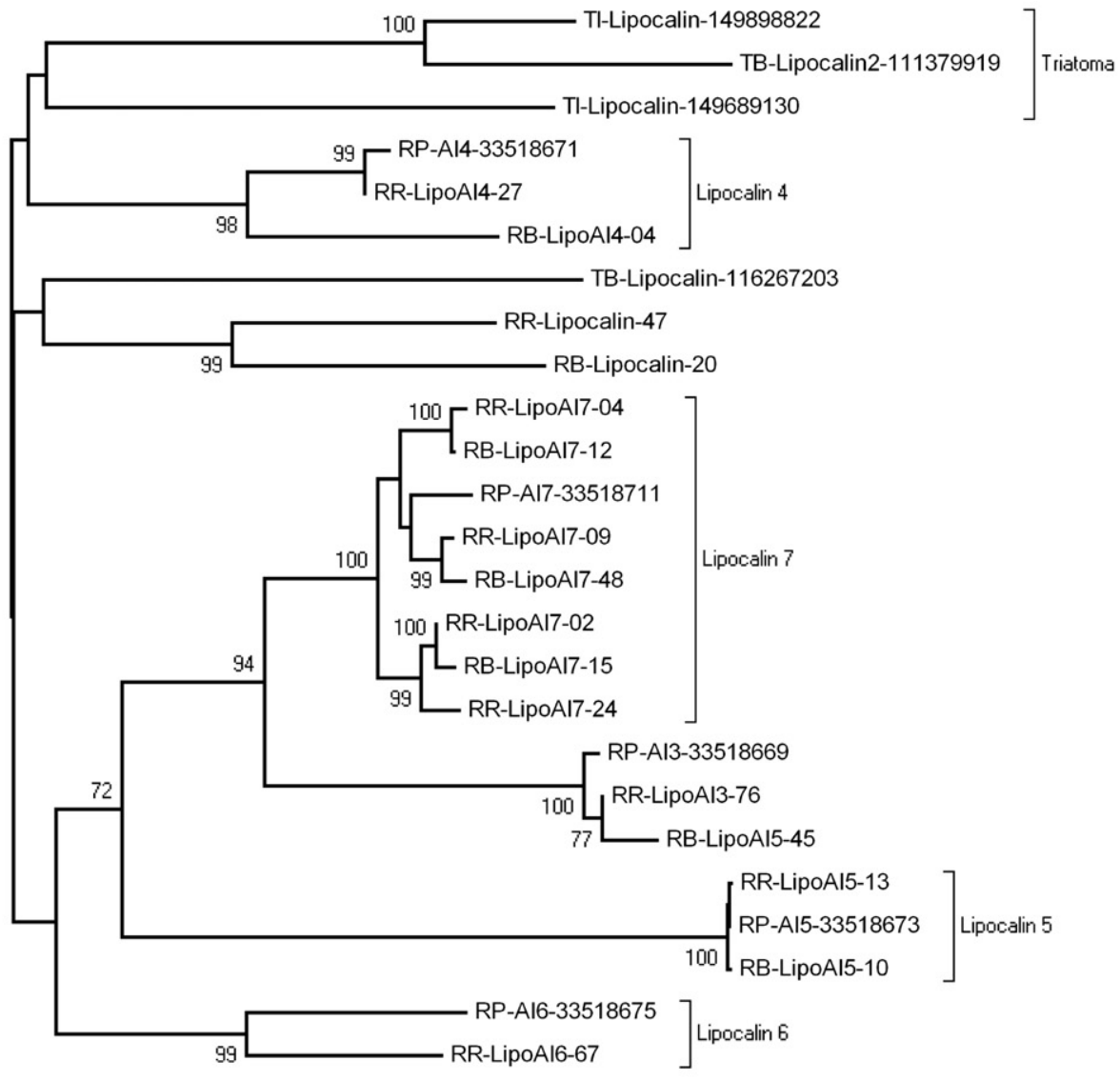

B

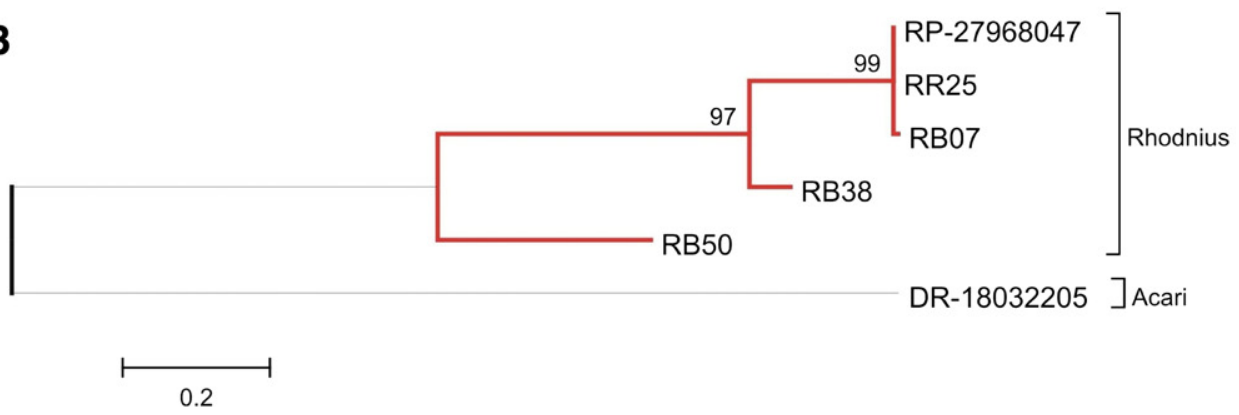

Fig. 2 - Comparative analyses of lipocalins and of biogenic amine binding proteins in the salivary glands of Rhodnius brethesi and of Rhodnius robustus. (A) Lipocalins dendogram from Triatoma infestans (TI), Triatoma brasiliensis (TB), Rhodnius prolixus (RP), Rhodnius brethesi (RB) and Rhodnius robustus (RR). The compared sequences are from the nonreduntant protein database of the National Center for Biotechnology Information (NCBI) and are represented by the first letters of gender and specie followed by the NCBI gi| accession number. The numbers in the dendogram nodes indicate percent bootstrap support for the phylogeny. The bar (bottom) indicates $20 \%$ amino acid divergence in the sequences. The graphic was constructed with MEGA4 package and the cut-off 70 was observed [18]; (B) Phylogram of biogenic amine binding protein and related proteins. 

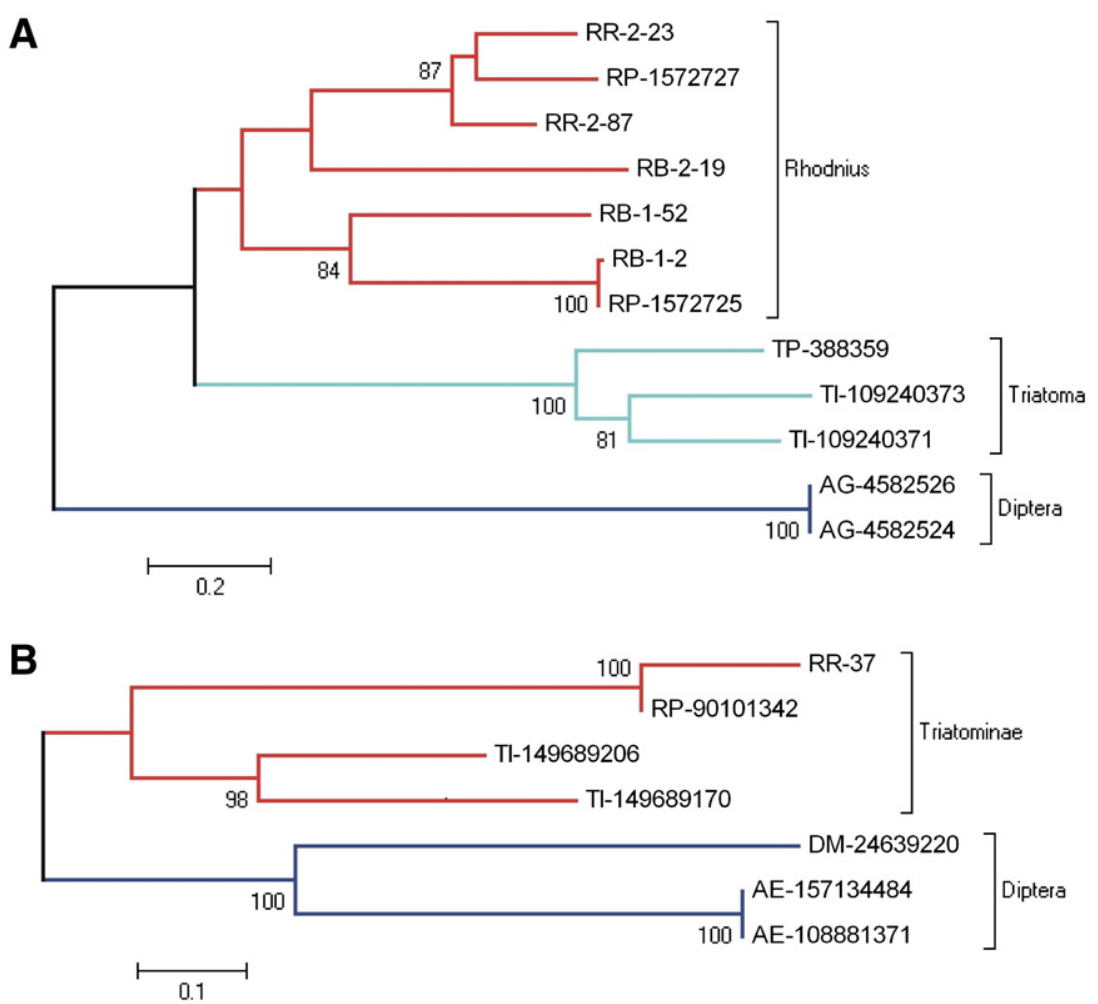

Fig. 3 - Comparative analysis of platelet aggregation inhibitor and of inositol polyphosphate 5-phosphatase proteins in the salivary glands of $R$. brethesi and R. robustus. (A) Dendogram of salivary platelet aggregation inhibitor (SPAI) proteins with R. prolixus (RP), Triatoma infestans (TI), Triatoma pallidipennis (TP), Anopheles gambiae (AG), R. brethesi (RB) and R. robustus (RR); (B) Phylogram of inositol polyphosphate 5-phosphatase and related proteins.

T. infestans, T. brasiliensis and R. prolixus, which were divided in lipocalins subtypes 4, 5, 6, and 7 .

The nitrophorins are important members of lipocalins family, which are nitric oxide (NO)-binding proteins. NO is used in the host organism as a signaling molecule, which is produced and released by the vascular endothelium; it activates the soluble guanylate cyclase, leading to the muscle relaxation [28]. NO appears to be released during transportation by the bug's nitrophorins, depending on the local pH [29]. In low $\mathrm{pH}$ of the salivary gland lumen, the NO is bound to the heme protein, whereas in the host organism the increase of the $\mathrm{pH}$ releases the NO with induction of vasodilation. Phylogenetic tree of the nitrophorins family shows close relationship between nitrophorins 1 and 4 and proximity to nitrophorins 2 and 3, as expected $[27,28]$.

\subsubsection{Biogenic amine-binding proteins (BABP)}

One BABP transcript was found in the cDNA library of $R$. robustus and three transcripts in $R$. brethesi; the alignment of those sequences (Fig. 2B) showed similarity with BABP from $R$. prolixus. The transcript $R R-25$ had the same cysteine residues present in $R$. prolixus BABP, where four amino acids formed disulfide bridges. The dendogram of Rhodnius species clustered in a branch, whereas a BABP protein from Dermacentor reticulatus, showing considerable phylogenetic distance was clustered in a different branch (Fig. 2C).

BABPs are part of the lipocalin family. Those proteins bind to serotonin and histamine to prevent their dispersion in the blood vessels. The absence of BABP favors insect's bloodsucking a plain meal. A BABP from R. prolixus, which binds to serotonin, epinephrine, norepinephrine, was shown to inhibit muscle contractions [30]. BABP retained serotonin and epinephrine agonists from the platelet activation pathway, and showed a platelet aggregation inhibitor activity. R. prolixus BABP binding pro-inflammatory molecules inhibited the inflammatory response [30].

\subsubsection{Salivary platelet aggregation inhibitors (SPAI)}

Two transcripts from $R$. robustus and three from $R$. brethesi were classified as platelet aggregation inhibitor. The phylogenetic analysis (Fig. 3A) showed their proximity with the $R$. prolixus transcripts. A Rhodnius prolixus aggregation inhibitor, which is similar to pallidipin from $T$. pallidipennis uses adenosine diphosphate (ADP) in low concentration and prevents further platelet activation [14]. The platelet aggregation inhibitors can be activated immediately after the insect's stylet enters the host skin. To prevent blood loss and repair the tissue damage, the insect triggers off the homeostasisdependent platelet aggregation, vasoconstriction and blood coagulation pathways. The host platelets activation by collagen, ADP, tromboxane A2, and thrombin interactions augments platelet aggregation and clot formation [31].

\subsubsection{Inositol polyphosphate 5-phosphatase (IPP)}

The IPP family in insects is composed by enzymes that remove phosphate from the 5-position of the inositol ring [32]. The $R$. 


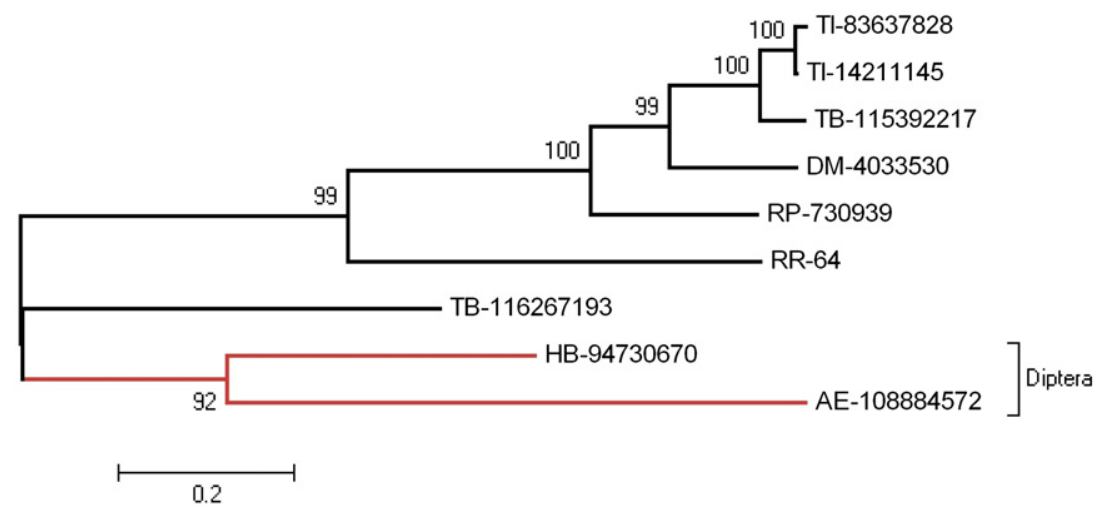

Fig. 4 - Comparative analysis of Kazal domain proteins in the salivary glands of R. brethesi and R. robustus. Phylogram showing Kazal domain of insects' proteins.

robustus IPP did not present the conserved motif FWLGDLNRFI and PSWTDRVLY. A polypeptide with similar feature was described in R. prolixus [9], and, instead the R. prolixus IPP shows a domain with preference for soluble and lipid substrates [32]. The divergence between hemiptera triatomines and other diptera insects is depicted in Fig. 3B. Phosphodiesterase enzyme cleaves cyclic nucleotides that regulate platelet aggregation and muscle contraction. The IPP is classified in seven different families, accordingly with substrate specificity [33].

\subsubsection{Kazal domain proteins}

The $R$. robustus had one sequence of Kazal domain protein similar to brasiliensin. The presence of six cysteine residues and two other aminoacids (TY) defined the Kazal domain gene family. The alignment generated phylogenetic network (Fig. 4) revealed a clear divergence between hemiptera triatomines and diptera. Interestingly, the thrombin inhibitors were close in D. maximus (dipetalogastin), T. brasiliensis (brasiliensin), $R$. prolixus (rhodniini), and $\mathrm{T}$. infestans (infestin and thrombin inhibitor). The Kazal proteins in hematophagous insects have different functions. A thrombin inhibitor was described in $R$. prolixus [34], which had 103 amino acid residues (11 kDa). The dipetalogastins in Dipetalogaster maximus midgut [35] was coded by a huge gene, which could express a protein with six Kazal-type domains, which main functions were thrombin and trypsin inhibitions. The T. brasiliensis thrombrin inhibitor 'brasiliensin' is coded by a gene with $8 \mathrm{Kazal}$ domains [36].

\subsubsection{Antigen 5-like protein}

The R. robustus transcriptome revealed a protein similar to Ag5 from $T$. infestans and R. prolixus with basic tails replenished of lysines. This feature could modify its interaction with active ligands [10]. Four Ag5 family proteins were demonstrated in $R$. brethesi and three of these were present in $R$. robustus proteomes. Antigen 5-like protein (Ag5) is part of a CAP protein family that encloses secreted sequences with similarity in a core of 150 amino acids [37]. Ag5 is the main venom of vespid wasp [38].

\subsubsection{Glutathione S-transferase and insect resistance}

The $R$. brethesi proteome revealed a single protein with similarity to GST from Anopheles dirus. Also GST that was described in T. infestans was associated to resistance to DTT [1,1,1-trichloro-2,2-bis(4-chlorophenyl)ethane] [10,39], and a similar gene was identified in R. prolixus [9]. Glutathione Stransferase (GST) belongs to an enzymatic family involved with detoxication. The enzyme catalyzes the conjugation of eletrophylic compound with thiol group of the reduced glutathione; the water soluble by-product can be easily excreted [39]. The microsomal and cytosolic GSTs are part of a multigenic family possibly resulting from single gene expression. This detoxication system in insects can augment the production of protective enzymes $[39,40]$.

\section{Conclusions}

The $R$. robustus and $R$. brethesi are main species of cone-nosed bugs transmitting the T. cruzi infections and current outbreaks of acute Chagas disease in the Amazon Basin. The scientific knowledge about the physiology and biochemical feeding patterns of these insect-vectors of the T. cruzi infections may contribute to control the epidemics of Chagas disease in the Amazon. In this regard, the high-throughput molecular transcriptomics and LC-MS/MS proteomic technologies revealed several unknown features related to blood-feeding of the triatomines. Here we showed that lipocalins are farfetched main rhodniini salivary protein in these Amazonian Rhodnius species. Additionally, inositol 5 phosphatase, Kazal domain proteins, detoxication protein (GST), and antigen5 proteins were disclosed, which had never been reported in these species. The molecular phylogenetics showed that $R$. brethesi and R. robustus close relatives to $R$. prolixus shared several lipocalins, whereas Triatoma infestans, which are placed apart in the phylum, did not. This multidisciplinary study is an attempt to understand triatomines complex salivary gland apparatuses that could lead to the discovery of inhibitors of key metabolic pathways crucial for preventing the insect's blood feeding and survival. Novel inhibitory molecules are required for formulation of repellents, aiming at prevention of the T. cruzi infections.

Supplementary materials related to this article can be found online at doi:10.1016/j.jprot.2011.06.022. 


\section{Acknowledgments}

The authors thank Adriana Xavier and Ana de Cassia Rosa for technical Assistance. We are indebted to Dr. Aldo Valente from the Evandro Chagas Institute, Belém, Pará, Brazil, for donation of the Rhodnius species captured in the Amazon. This work received financial support from the National Research Council/CNPq, Brazil, and from the National Institutes of Health (Grants 2G12RR008124-16A1 and 2G12RR00812416A1S1). We are grateful to the Biomolecule Analysis Core Facility/BBRC/UTEP (NIH grants 2G12RR008124-16A1 and 2G12RR008124-16A1S1) where the LC-MS/MS analyses were performed. ESN was partially supported by the George A. Krutilek Memorial Scholarship, Graduate School, University of Texas at El Paso.

Data availability

Raw LC-MS/MS data is freely available at Tranche database

(https://proteomecommons.org/) under hash:

oWsKKrcXgHqOjxSBlx5Zp2LfIwqsELpV09RE/QcJeJUfqYyIfC VZd5Wsj4RSbQ4zyuqn9/IwkOUHquJQxY3Vs0ToLLIAAAAAA $\mathrm{AACbA}==$

\section{R E F E R E N C E S}

[1] Abad-Franch F, Monteiro FA, Jaramillo N, Gurgel-Goncalves R, Braga F, Dias FB, et al. Ecology, evolution, and the long-term surveillance of vector-borne Chagas disease: a multi-scale appraisal of the tribe Rhodniini (Triatominae). Acta Trop 2009;110:159-77.

[2] Aguilar HM, Abad-Franch F, Dias JCP, Junqueira ACV, Coura JR. Chagas disease in the Amazon Region. Mem Inst Oswaldo Cruz 2007;102:47-55.

[3] Coura JR, Junqueira ACV, Boia MN, Fernandes O. Chagas disease: from bush to huts and houses. Is it the case of the Brazilian Amazon? Mem Inst Oswaldo Cruz 1999;94:379-84.

[4] Teixeira ARL, Monteiro PS, Rebelo JM, Argañaraz ER, Vieira D, Lauria-Pires L, et al. Emerging chagas disease: trophic network and cilce of transmission of Trypanosoma cruzi from palm trees in the Amazon. Emerg Infect Dis 2001;7:100-12.

[5] Valente SA, da Costa Valente V, das Neves Pinto AY, de Jesus Barbosa CM, dos Santos MP, Miranda CO, et al. Analysis of an acute Chagas disease outbreak in the Brazilian Amazon: human cases, triatomines, reservoir mammals and parasites. Trans R Soc Trop Med Hyg 2009;103:291-7.

[6] Pinto AY, Valente SA, Valente Vda C, Ferreira Junior AG, Coura JR. Acute phase of Chagas disease in the Brazilian Amazon region: study of 233 cases from Pará, Amapá and Maranhão observed between 1988 and 2005. Rev Soc Bras Med Trop 2008;41:602-14.

[7] Abad-Franch F, Monteiro FA. Biogeography and evolution of Amazonian triatomines (Heteroptera: Reduviidae): implications for Chagas disease surveillance in humid forest ecoregions. Mem Inst Oswaldo Cruz 2007;102:57-69.

[8] Champagne DE. Antihemostatic molecules from saliva of blood-feeding arthropods. Pathophysiol Haemost Thromb 2004;34:221-7.

[9] Ribeiro JMC, Andersen J, Silva-Neto MAC, Phama VM, Garfield M, Valenzuela JG. Exploring the sialome of the blood-sucking bug Rhodnius prolixus. Insect Biochem Mol Biol 2004;34:61-79.

[10] Assumpção TCF, Francischetti IMB, Andersen JF, Schwartz A, Santana JM, Ribeiro JM. An insight into the sialoma of the blood-sucking bug Triatoma infestans, a vector of Chagas' disease. Insect Biochem Mol Biol 2008;38:213-32.

[11] Santos A, Ribeiro JM, Lehane MJ, Gontijo NF, Veloso AB, Sant'Anna MR, et al. The sialotranscriptome of the blood-sucking bug Triatoma brasiliensis (Hemiptera, Triatominae). Insect Biochem Mol Biol 2007;37:702-12.

[12] Gutierrez G, Ganfornina MD, Sanchez D. Evolution of the lipocalin family as inferred from a protein sequence phylogeny. Biochem Biophys Acta 2000;1482:35-45.

[13] Charneau S, Junqueira M, Costa CM, Pires LP, Fernandes ES, Bussacos AC, et al. The saliva proteome of the blood-feeding insect Triatoma infestans is rich in platelet-aggregation inhibitors. Int J Mass Spectrom 2007;268:265-76.

[14] Francischetti IMB, Ribeiro JMC, Champagne D, Andersen J. Purification, cloning, expression, and mechanism of action of a novel platelet aggregation inhibitor from the salivary gland of the blood-sucking bug, Rhodnius prolixus. J Biol Chem 2000;275:12639-50.

[15] Altschul SF, Gish W. Local alignment statistics. Methods Enzymol 1996;266:460-80.

[16] Thompson JD, Higgins DG, Gibson TJ. CLUSTAL W: improving the sensitivity of progressive multiple sequence alignment through sequence weighting, position-specific gap penalties and weight matrix choice. Nucleic Acids Res 1994;22:4673-80.

[17] Marchler-Bauer A, Anderson JB, Derbyshire MK, DeWeese-Scott C, Gonzales NR, Gwadz M, et al. CDD: a conserved domain database for interactive domain family analysis. Nucleic Acids Res 2007;35:237-40.

[18] Page RD. TreeView: an application to display phylogenetic trees on personal computers. Comput Appl Biosci 1996;12:357-8.

[19] Tamura K, Dudley J, Nei M, Kumar S. MEGA4: Molecular Evolutionary Genetics Analysis (MEGA) software version 4.0. Mol Biol Evol 2007;24:1596-9.

[20] Rodrigues ML, Nakayasu ES, Oliveira DL, Nimrichter L, Nosanchuk JD, Almeida IC, et al. Extracellular vesicles produced by Cryptococcus neoformans contain protein components associated with virulence. Eukaryot Cell 2008;7:58-67.

[21] Eng JK, McCormack AL, Yates III JR. An approach to correlate tandem mass spectral data of peptides with amino acid sequences in a protein database. J Am Soc Mass Spectrom 1994;5:976-89.

[22] Francischetti IM, Valenzuela JG, Pham VM, Garfield MK, Ribeiro JM. Toward a catalog for the transcripts and proteins (sialome) from the salivary gland of the malaria vector Anopheles gambiae. J Exp Biol 2002;205:2429-3451.

[23] Francischetti IMB, Andersen JF, Ribeiro JMC. Biochemical and functional characterization of recombinant Rhodnius prolixus platelet aggregation inhibitor 1 as a novel lipocalin with high affinity for adenosine diphosphate and other adenine nucleotides. Biochemistry 2002;41:3810-8.

[24] Andersen JF, Gudderra NP, Francischetti IMB, Ribeiro JMC. The role of salivary lipocalins in blood feeding by Rhodnius prolixus. Arch Insect Biochem Physiol 2005;58:97-105.

[25] Flower DR. The lipocalin protein family: structure and function. Biochem J 1996;318:1-14.

[26] Flower DR, North ACT, Sansom CE. The lipocalin protein family: structural and sequence overview. Biochem Biophys Acta 2000;1482:9-24.

[27] Grzyb J, Latowski D, Strzalka K. Lipocalins - a family portrait. J Plant Physiol 2006;163:895-915.

[28] Andersen JF, Champagne DE, Weichsel A, Ribeiro JM, Balfour CA, Dress V, et al. Nitric oxide binding and crystallization of recombinant nitrophorin I, a nitric oxide transport protein from the blood-sucking bug Rhodnius prolixus. Biochemistry 1997;36:4423-8.

[29] Montfort WR, Weichsel A, Andersen JF. Nitrophorins and related antihemostatic lipocalins from Rhodnius prolixus and other blood-sucking arthropods. Biochem Biophys Acta 2000;1482:110-8. 
[30] Andersen JF, Francischetti IMB, Valenzuela JG, Schuck P, Ribeiro JMC. Inhibition of hemostasis by a high affinity biogenic amine-binding protein from the saliva of a blood-feeding insect. J Biol Chem 2003;278:4611-7.

[31] Noeske-Jungblut C, Krätzschmar J, Haendler B, Alagon A, Possani L, Verhallen P, et al. An inhibitor of collagen-induced platelet aggregation from the saliva of Triatoma pallidipennis. J Biol Chem 1994;269:5050-3.

[32] Andersen JF, Ribeiro JMC. A secreted salivary inositol polyphosphate 5-phosphatase from a blood-feeding insect: allosteric activation by soluble phosphoinositides and phosphatidylserine. Biochemistry 2006;45:5450-7.

[33] Erneux C, Govaerts C, Communi D, Pesesse X. The diversity and possible functions of the inositol polyphosphate 5-phosphatases. Biochim Biophys Acta 1998;1436:185-99.

[34] Friedrich T, Kröger B, Bialojan S, Lemaire HG, Höffken HW, Reuschenbach P. A Kazal-type inhibitor with thrombin specificity from Rhodnius prolixus. J Biol Chem 1993;268: 16216-22.

[35] Mende K, Lange U, Nowak G. Three recombinant serine proteinase inhibitors expressed from the coding region of the thrombin inhibitor dipetalogastin. Insect Biochem Mol Biol 2004;34:971-9.

[36] Araujo RN, Campos IT, Tanaka AS, Santos A, Gontijo NF, Lehane MJ, et al. Brasiliensin: a novel intestinal thrombin inhibitor from Triatoma brasiliensis (Hemiptera: Reduviidae) with an important role in blood intake. Int J Parasitol 2007;37:1351-8.

[37] Megraw T, Kaufman TC, Kovalick GE. Sequence and expression of Drosophila Antigen 5-related 2, a new member of the CAP gene family. Gene 1998;222:297-304.

[38] Hoffman DR. Allergens I hymenoptera venom XXV: the amino acid sequences of antigen 5 molecules and the structural basis of antigenic cross-reactivity. J Allergy Clin Immunol 1993;92:707-16.

[39] Enayati AA, Ranson H, Hemingway J. Insect glutathione transferases and insecticide resistance. Insect Mol Biol 2005;14:3-8.

[40] Sívori JL, Casabé N, Zerba EN, Wood EJ. Induction of glutathione S-transferase activity in Triatoma infestans. Mem Inst Oswaldo Cruz 1997;92:797-802. 